\title{
Taking up the challenge: Can effective long-term intra-uterine contraceptive methods radically reduce the number of unintended pregnancies?
}

In his remarkable article entitled 'Contraceptive prevalence, reproductive health and our common future', published in 1990, Diczfalusy wrote: 'The 1980s will go into history as a decade of lost opportunities to increase contraceptive prevalence and improve reproductive health world-wide. As the decade closes, 500 million couples still have no access to fertility regulation, there are 30-50 million induced abortions each year, 15 million infant and child deaths (30\% of all deaths world-wide), an estimated 250 million new cases of sexually transmitted diseases and 60-80 million infertile couples'. ${ }^{1}$

Over the last 10 years the world has changed considerably. In many respects, the situation is far worse than 10 years ago. The UN estimated recently that the population is growing by about 78 million per year. Almost all population growth is in the developing world, and about three quarters is urban. As cities grow ever larger, their impact on the environment grows exponentially.

Recent population studies have established the alarming increase of teenage pregnancies world-wide. We are facing the largest ever generation of young people entering adulthood. Millions of women begin their childbearing in their teens, mostly out of marriage. Earlier sexual maturity, pre-marital sex, later marriage and urban expansion contribute to the explosion of teenage pregnancy rates. The problem is huge since the majority of these pregnancies are unplanned and unintended. Some figures speak for themselves: more than $50 \%$ of pregnancies in the USA are unplanned; half of them (1.4 million per year) end in termination, of which over $50 \%$ are in women younger than 25 years of age, and $22 \%$ in adolescents. In Western Europe, the figures are similar; in the UK, in France and Italy there are roughly 200000 abortions yearly and $25 \%$ of the women are between 16 and 19 years of age.

Teenage pregnancy is unquestionably a world-wide problem: $58 \%$ of all mothers in sub-Saharan Africa are teenagers. A similar situation is seen in the Philippines, Thailand, India, Pakistan, Bangladesh and Central America. ${ }^{2}$ In China, the number of unintended pregnancies and abortions in teenagers has sharply increased during recent years. As many as 4.4 million abortions may be sought by adolescent girls each year. A large number of these abortions are clandestine and therefore unsafe.

The majority of unintended pregnancies are the consequence of lack of access to information and services, unwanted sexual relations, unprotected sex, or ineffective use of contraception. The latter can result from providing too few options, inadequate information or unsuitable methods for certain subgroups of teenage women. In spite of the widescale availability of the pill (at least in the Western world) and the significant progress in contraceptive technology which has been made in the past 40 years, there has been no reduction in unintended pregnancies in the past decade. The typical failure rate of the pill is still unacceptably high at 5\% due to inconsistent use and discontinuation. ${ }^{3}$ Between $40 \%$ and $60 \%$ of new pill users discontinue the pill during the first year. The average duration of use of the pill in the USA is only 4.8 months. The same phenomenon has been observed in Western Europe, where 50\% of adolescents stop using the pill after 3 months. It seems extremely hard for very young women to use the method correctly and consistently. It follows that contraceptive method failure rates, for methods which depend on user compliance, may be calculated incorrectly and be reported lower than reality.

It is clear that methods which are dependent on memory and motivation, such as the pill, are not the ideal solution in the younger age groups. For years, the pill has been synonymous with contraception. This has regrettably helped to maintain ignorance of any alternatives beyond condoms and sterilisation, although acceptable alternatives have demonstrated their superior effectiveness. With injectables, implants and IUDs, the inherent efficacy is so high, and proper and consistent use is almost guaranteed, that studies invariably demonstrate extremely low pregnancy rates. In a comparative study in 100 postpartum adolescents, of whom 50 selected the pill and 50 an implantable method (Norplant), one young mother in the Norplant group and 19 in the pill group became pregnant during the first postpartum year. ${ }^{4}$ It appears that the most effective method for an individual woman or couple is a method which minimises the risk of imperfect use. In women using a method inconsistently, the cumulative risk of pregnancy during lifetime is high. An annual probability of pregnancy of $3 \%$ implies a $26 \%$ probability of pregnancy over 10 years.

What can be done? One of the great obstacles to preventing unplanned pregnancy is the lack of access to more effective methods of contraception. We urgently need effective and affordable methods on a large scale that can be forgotten once initiated, and are usable 'first-line' by adolescents. ${ }^{5}$ Longacting injectables, implants, IUDs and hormone-releasing intra-uterine systems are methods which point the way forward, even if it takes time to learn how to insert them. A major advantage of long-acting hormonal methods is that they eliminate the need for specific action at the time of coitus, such as putting on a condom, or for daily action, such as the pill. They offer discretion and privacy. Unfortunately, some of them also have disadvantages because they disrupt the menstrual cycle causing breakthrough bleeding, amenorrhea or occasionally heavier bleeding. They can also cause systemic hormonal side effects.

Intra-uterine devices and intra-uterine systems are particularly attractive as they have the advantage of acting locally, avoiding systemic effects. They have less impact on menstrual pattern after the first few months and, when low dose levonorgestrel intra-uterine systems are used, they are less likely to cause initial spotting, amenorrhea and hormonal side-effects. New developments in intra-uterine technology are providing smaller frameless devices and devices that combine the features of a frameless copper device with a levonorgestrel system. They may be ideal for use in younger women because they are small, effective and well tolerated. Unlike the pill, they are genuinely 'fit-and-forget'. In use, they are much more effective than pills in this age group (see further reading). Moreover, they are long-acting and reversible. So, the reward is substantial. However, copper intra-uterine devices do not offer protection against sexually 
transmitted infections (STIs) and, therefore, they are not always the methods of first choice for teenagers. Such a protective effect has been observed with hormone-releasing intra-uterine devices in women aged 25 and under, although this finding was not confirmed in other studies. ${ }^{6}$ Nevertheless, in the current situation, they should be offered more frequently as first or second line methods, in combination with condoms if required, particularly after the first unintended pregnancy has occurred.

The World Health Organisation (WHO) supports the use of appropriate intra-uterine methods in young women and suggests that the benefits of intra-uterine contraceptives generally outweigh the risks in women of any age, whether or not they have had children. In addition, WHO approves the use of these methods in women under 20 years of age, provided that they are at low risk of sexually transmitted infections. A recent re-assessment of the risk of pelvic inflammatory disease attributable to an intra-uterine device suggested that the estimated risk was low, only 0.15 , even in regions where the prevalence of STIs is high. ${ }^{7}$ Drug delivery systems which impact on the cervix could reduce the potential for upper genital tract infection further. This could be a task for future generations of scientists - to focus on contraceptive developments that will also provide solutions to the ever-increasing problem of sexual transmission of infection. In the meantime, using two methods at once reduces the probability of pregnancy significantly while adding protection from disease transmission.

All couples should be able to consider, without coercion, the responsibilities which attach to the creation of a child; they should have access to effective, affordable and trouble-free means to prevent the conception of unwanted children and they should retain the ability to restore normal fertility and have children when they are ready and able to care for them.
Whether or not we will be able to make progress and reach our objectives will depend largely on non-scientific factors. National decision-makers should realise that contraceptive prevalence represents the key not only to improved reproductive and environmental health, but also to demographic and economic development.

\section{Statements on funding and competing interests}

Funding. None declared.

Competing interests. Dirk Wildemeersch is a Belgian gynaecologist and Medical Director of Contrel Research, a company which was established to manage clinical research and to develop and study innovative drug delivery technologies, aimed at finding improved methods for prevention and treatment of gynaecological conditions, improvements to birth control methods, and higher levels of safety, user acceptability, compliance and quality of life for women. Contrel is the manufacturer of GyneFix and FibroPlant. The funds generated are re-invested in research.

\section{Dirk Wildemeersch}

Gynaecologist, Piers de Raveschootlaan 125, 8300 Knokke-Heist, Belgium. Tel: +32 50600 900, Fax: +32 50 622 429, Email: dirk.wildemeersch@contrel.be, Website: www.contrel.be

References

Diczfalusy E. Contraceptive prevalence, reproductive health and our common future. Contraception 1990; 43: 201-227.

UNFPA. The State of World Population Growth 1998. United Population Fund. ISBN 0-89714444-9 E/31,000/1998.

Trussell J. Contraceptive efficacy. In: Hatcher RA, Trussell J, Stewart F, et al (Eds). Contraceptive Technology: Seventeenth Revised Edition. New York, NY: Ardent media, 1998. , et al. The use of levonorgestrel implants (Norplant) for contraception in adolescent mothers. N Engl J Med 1994, 331(18): 1201-1230.

Guillebaud J. Looking to the future. In: Ferguson J, Upsdell M (Eds) Key Advances in the Effective Management of Contraception. London: Royal Society of Medicine Press, 1999:

Andersson K, Odlind V, Rybo G. Levonorgestrel-releasing and copper-releasing (Nova T) IUD during five years of use: A randomized comparative tral. Contraception 1994; 49: 56-72. 357: 443 